This item is the archived peer-reviewed author-version of:

\title{
Homogeneity and composition of AllnGaN : a multiprobe nanostructure study
}

\section{Reference:}

Krause Florian F., Ahl Jan-Philipp, Tytko Darius, Egoavil Escobar Ricardo Juan, Verbeeck Johan, et al..- Homogeneity and composition of AllnGaN : a multiprobe nanostructure study

Ultramicroscopy - ISSN 0304-3991 - 156(2015), p. 29-36

DOI: http://dx.doi.org/doi:10.1016/j.ultramic.2015.04.012 


\title{
Homogeneity and Composition of AlInGaN: A Multiprobe Nanostructure Study
}

\author{
Florian F. Krause ${ }^{\mathrm{a}, *}$, Jan-Philipp Ahl ${ }^{\mathrm{b}}$, Darius Tytko ${ }^{\mathrm{c}}$, Pyuck-Pa Choi ${ }^{\mathrm{c}}$, \\ Ricardo Egoavil ${ }^{\mathrm{d}}$, Marco Schowalter ${ }^{\mathrm{a}}$, Thorsten Mehrtens ${ }^{\mathrm{a}}$, Knut \\ Müller-Caspary ${ }^{\mathrm{a}}$, Johan Verbeeck ${ }^{\mathrm{d}}$, Dierk Raabe ${ }^{\mathrm{c}}$, Joachim Hertkorn ${ }^{\mathrm{b}}$, Karl \\ Engl $^{\mathrm{b}}$, Andreas Rosenauer ${ }^{\mathrm{a}}$ \\ ${ }^{a}$ Institut für Festkörperphysik, Universität Bremen, Otto-Hahn-Allee 1, 28359 Bremen, \\ Germany \\ ${ }^{b}$ OSRAM Opto Semiconductors GmbH, Leibnizstr. 4, 93055 Regensburg, Germany \\ ${ }^{c}$ Max-Planck-Institut für Eisenforschung GmbH, Max-Planck-Str. 1, 40237 Düsseldorf , \\ Germany \\ ${ }^{d}$ EMAT, Universiteit Antwerpen, Groenenborgerlaan 171, 2020 Antwerpen, Belgium
}

\begin{abstract}
The electronic properties of quaternary AlInGaN devices significantly depend on the homogeneity of the alloy. The identification of compositional fluctuations or verification of random-alloy distribution is hence of grave importance. Here, a comprehensive multiprobe study of composition and compositional homogeneity is presented, investigating AlInGaN layers with indium concentrations ranging from 0 to 17 at.\% and aluminum concentrations between 0 and 39 at.\% employing high-angle annular dark field scanning electron microscopy (HAADF STEM), energy dispersive X-ray spectroscopy (EDX) and atom probe tomography (APT). EDX mappings reveal distributions of local concentrations which are in good agreement with random alloy atomic distributions. This was hence investigated with HAADF STEM by comparison with theoretical random alloy expectations using statistical tests. To validate the performance of these tests, HAADF STEM image simulations were carried out for the case of a randomalloy distribution of atoms and for the case of In-rich clusters with nanometer dimensions. The investigated samples, which were grown by metal-organic vapor phase epitaxy (MOVPE), were thereby found to be homogeneous on this nanometer scale. Analysis of reconstructions obtained from APT measurements yielded matching results. Though HAADF STEM only allows for the reduction of possible combinations of indium and aluminum concentrations to the proximity of isolines in the two-dimensional composition space. The observed ranges of composition are in good agreement with the EDX and APT results within the respective precisions.
\end{abstract}

Keywords: HAADF STEM, AlInGaN, Homogeneity, InAlGaN, EDX, APT

\footnotetext{
* Corresponding author

Email address: f.krause@ifp.uni-bremen.de (Florian F. Krause)
} 


\section{Introduction}

Quaternary AlInGaN has attracted increasing attention in the past years for the design of blue light emitting diodes (LEDs) [1, 2] and field-effect transistors (FETs) [3, 4]. The material benefits from its additional degree of freedom opposed to ternary systems, which to a certain extent allows an independent choice of either bandgap and lattice constant, allowing for the realisation of lattice-matched structures, or bandgap and polarisation, enabling so-called polarisation-matched growth of AlInGaN/GaN heterostructures. In these structures, polarisation effects such as the quantum-confined Stark effect are reduced, which is expected to lead to an increase in efficiency [5].

Besides the matching of polarisation, homogeneity of the alloy as well as defect density and morphology of the interfaces govern the electronic properties of AlInGaN-based devices [6-9]. Spectral width and efficiency of the emission of light from quantum wells (QWs) strongly depend on the distribution of indium, aluminum and gallium atoms within the wells. Some publications reported observations of indium-rich cluster formation, catalysed by the presence of aluminum in the quaternary material opposed to ternary InGaN [10, 11]. Matsuoka [12] even found an unstable mixing region covering most of the quaternary composition space, whereas Marques et al. [13] predicted a phase transition from clustering to homogeneous mixing behavior at $\approx 800^{\circ} \mathrm{C}$ growth temperature. The present work demonstrates a comprehensive investigation of compositional homogeneity in AllnGaN layers grown by metal-organic vapour phase epitaxy (MOVPE) employing different high-resolution experimental methods, specifically, energy dispersive X-ray spectroscopy (EDX), high-angle annular dark field scanning transmission electron microscopy (HAADF STEM) and atomprobe tomography (APT).

HAADF STEM has already successfully been used for the investigation of composition and compositional homogeneity in ternary materials such as AlGaN, InGaN or InAlN [14-16]. It benefits from the reduced beam damage compared to parallel illumination [15, 17]. For the quaternary material $\mathrm{Al}_{x} \operatorname{In}_{y} \mathrm{Ga}_{1-x-y} \mathrm{~N}$, however, measurement of indium and aluminum concentrations would require two separate inequivalent measured quantities. Thus, HAADF STEM measurements alone do not allow for determination of the composition in AlInGaN.

EDX, however, which is available during STEM measurements in modern electron microscopes, makes the simultaneous determination of both concentrations possible. With high brightness electron sources, probe correctors and windowless four-quadrant X-ray detectors it can even reach atomic resolution today [18]. For the investigation of possible clustering on the nanometer scale, however, long acquisition times are necessary to reach sufficient statistical significance, during which specimen drift can occur, cumbering the statistical analysis. Furthermore, as the resulting composition map is a two-dimensional projection 
of the specimen, in general corresponding simulations would be necessary to understand the exact volume of interaction in dependence of probe position.

APT-investigations overcome both disadvantages, as in these reconstructions both, the type and the initial position of each detected ion are determined, allowing for a three-dimensional investigation of the spatial distribution of all species with respect to all other species. The crystal volume which is accessible in one measurement is, however, limited even compared to the nanometer-thin TEM-lamellas due to the sharp needle shape of the APT specimen.

In the following, a comprehensive study of composition and homogeneity of AlInGaN with various concentrations using all methods mentioned above is presented. Samples with nominal compositions as listed in Tab. 1, comprising layers with four different aluminum concentrations between 0 and 39 at.\% and 12 at.\% In as well as AlGaN layers with nominal 39 at.\% Al were investigated.

\section{Specimens and Instrumentation}

The specimen primarily investigated here consists of 5 consecutive layers of AlInGaN of different concentrations embedded in GaN. It was grown by MOVPE on GaN/sapphire templates. Each layer is $\approx 10 \mathrm{~nm}$ thick and is separated from the next layer by a barrier of $\approx 50 \mathrm{~nm}$ GaN. They were grown far from thermodynamic equilibrium [19] at a temperature of $760{ }^{\circ} \mathrm{C}$ and a pressure of $200 \mathrm{hPa}$. A more detailed description of the growth and investigation of the surface morphology is given in the publication of Ahl et al. [20]. Two of the layers consist of InGaN and AlGaN, respectively, providing ternary references for the investigations. Single-layer specimens with thicker layers but with the same nominal compositions were grown under identical conditions. These samples were used for the EDX measurements as they provide larger areas for simultaneous data acquisition within rectangular shaped areas of interest.

The first four compositions in Tab. 1 were pre-characterised by X-ray photoelectron spectroscopy (XPS) and photoluminescence spectroscopy (PL) [20]. The concentrations determined by XPS are also given in Tab. 1, whereas the spectra gained from the PL-measurement are depicted in Fig. 1. Besides the expected blue-shift for increasing aluminum concentrations, a broadening of the quaternary luminescence peak is observed. In the present paper, it is investigated, whether this increasing width is solely caused by the local concentration fluctuations inevitably occurring in a random alloy, which increase with the number of different constituents, or whether in addition indium clustering contributes to this effect.

A very thin sample was conventionally prepared by mechanical grinding and subsequent ion polishing for HAADF STEM, whereas for the EDX analyses, lamellas were prepared with a focused ion beam (FIB) using the lift-out method [21]. The samples were afterwards milled with low-energy Ar ions at $400 \mathrm{eV}$ to remove etching damage [22]. The needle-shaped tip specimens for APT measurements were likewise prepared with a FIB using the lift-out method described in Ref. [23] and sharpened while successively reducing the ion energy from $30 \mathrm{kV}$ to $2 \mathrm{kV}$. 
Table 1: Nominal aluminum and indium concentrations of the five investigated compositions and the values measured by the different methods. The given errors were estimated from consecutive measurements of the same sample.

\begin{tabular}{|l||c|c|c|c|c|c|c|c|}
\hline \multirow{2}{*}[\text{at.\%}]{} & nom. & \multicolumn{2}{|c|}{ XPS $[20]$} & \multicolumn{2}{c|}{ EDX } & \multicolumn{2}{c|}{ APT } \\
\cline { 2 - 9 } & $\mathrm{Al}$ & In & $\mathrm{Al}$ & $\mathrm{In}$ & $\mathrm{Al}$ & $\mathrm{In}$ & $\mathrm{Al}$ & $\mathrm{In}$ \\
\hline \hline no.1 & 0 & 12 & $0 \pm 0$ & $13 \pm 1$ & $0 \pm 0$ & $10 \pm 4$ & $0 \pm 0$ & $12 \pm 2$ \\
\hline no.2 & 13 & 12 & $13 \pm 1$ & $18 \pm 1$ & $17 \pm 3$ & $14 \pm 3$ & $16 \pm 2$ & $16 \pm 2$ \\
\hline no.3 & 22 & 12 & $22 \pm 1$ & $20 \pm 1$ & $23 \pm 3$ & $16 \pm 3$ & $25 \pm 2$ & $16 \pm 2$ \\
\hline no.4 & 39 & 12 & $39 \pm 1$ & $19 \pm 1$ & $33 \pm 3$ & $14 \pm 3$ & $31 \pm 2$ & $17 \pm 2$ \\
\hline no.5 & 39 & 0 & - & - & $39 \pm 3$ & $0 \pm 0$ & $36 \pm 2$ & $0 \pm 0$ \\
\hline
\end{tabular}

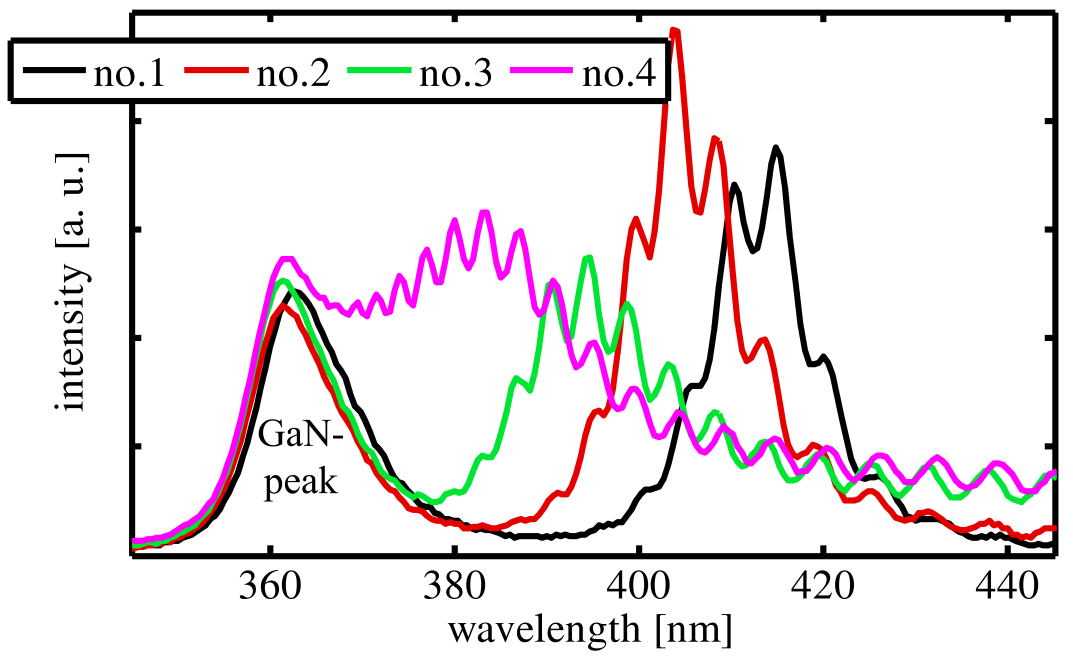

Figure 1: PL-spectra of compositions no.1 to 4: Besides a blue-shift of the quaternary peak with rising aluminum content an increase of the peak-width can be observed. While the ternary material no. 1 exhibits a full width at half maximum of $10 \mathrm{~nm}$ this increases to $\approx 27 \mathrm{~nm}$ for the composition with the highest aluminum content. The oscillations for wavelengths above to the GaN peak are caused by Fabry-Perot interference in the GaN substrate. 
EDX-maps were recorded with the XAntEM microscope of the University of Antwerp, a cubed FEI Titan ${ }^{3}$ 60/300 G2 TEM with ChemiSTEM-design equipped with a high-brightness X-FEG, a probe aberration corrector and a windowless Super-X EDX-detector, which was operated at $300 \mathrm{kV}$. The HAADF STEM investigations were performed at a FEI Titan 80/300 G1 microscope also operated at $300 \mathrm{kV}$ and equipped with a Fischione Model 3000 HAADF detector spanning from 30 to approximately $250 \mathrm{mrad}$. Atom probe tomography was conducted using a Cameca LEAP 3000X HR instrument.

\section{Results and Discussion}

\subsection{EDX-Investigation}

First, the single-layer specimens with layers of the nominal compositions given in Tab. 1 and a thickness of $(20 \pm 5) \mathrm{nm}$ were investigated by EDX. All samples were prepared as lamellas with a thickness of $(40 \pm 10) \mathrm{nm}$. EDX maps were acquired by drift-corrected, repetitive scanning of an area as shown in Fig. 2a for the quaternary specimen with the highest nominal aluminum concentration (no.4 in Tab. 1), for which inhomogeneities are most likely to occur $[12,13]$. The scan time was chosen 10 min per spectrum-image with a pixel width of $6 \AA$ to obtain a sufficiently high detection count rate for accurate quantification. For this the Cliff-Lorimer method [24] implemented in the proprietary Bruker ESPRIT software was used to evaluate the K-Lines of aluminum and gallium and the indium L-line with calculated k-factors of 1.00, 2.11 and 3.05, respectively, while the background subtraction was done with a polynomial fit from chosen background windows.

The resulting mean concentrations from averaging over the entire layer area are presented in Tab. 1, showing a generally good agreement with the nominal values. Fig. $2 \mathrm{~b}$ displays the resulting composition map for the aluminum-rich sample with composition no.4, which reveals an apparently homogeneous distribution of $\mathrm{In}, \mathrm{Al}$ and $\mathrm{Ga}$. To investigate the distributions quantitatively, histograms of the relative frequencies of local $\mathrm{In}, \mathrm{Al}$ and $\mathrm{Ga}$ concentrations were generated from the composition maps. They are shown in Fig. 2c for the map in Fig. 2b and for the ternary InGaN layer (composition no.1) in Fig. 2d for comparison. Using the mean concentration values determined by EDX in Tab. 1 as probabilities to detect $\mathrm{In}, \mathrm{Al}$ and $\mathrm{Ga}$ atoms, a theoretical distribution of concentrations was calculated by 100 independent random draws per pixel, each of which results in the detection of either indium, aluminum or gallium. The number of draws was chosen to match the ternary sample best. It roughly corresponds to the number of atoms stacked in an atomic column at this specimen thickness. The binomial distributions, which represent ideal homogeneity due to the mutual independence of the draws, are shown in Figs. 2c and $\mathrm{d}$ as solid curves. Both samples show good agreement between measured and binomial distributions, no increased deviation is observed for the quaternary specimen. Admittedly the assumption of a binomial distribution and especially

the reasoned but still somewhat arbitrary choice of 100 draws, complicates the 
quantification of homogeneity from these data. For a less empirical model better knowledge of the interaction volume of the electron beam within the specimen, of the X-ray propagation through the specimen and of the influence of detection and spectrum-quantification procedures would be required. However, the good representation of the experiment by the binomial model and the agreement being equally good for both the ternary and the quaternary sample, are clear indications for a homogeneous alloy, though the meaningfulness is limited due to the mentioned lack of knowledge. Analogous evaluations were executed for both other quaternary concentrations in Tab. 1 yielding similar results.

\subsection{HAADF STEM Study}

To further investigate the homogeneity of the AlInGaN alloys by statistical means, HAADF STEM images of AlInGaN crystals were simulated for various thicknesses with 31 at.\% $\mathrm{Al}$ and 16 at.\% In, corresponding to composition no.4 in Tab. 1, where the metal atoms were set according to a completely random distribution. The simulations were conducted using the STEMsim software package [25] with the frozen-lattice multislice method [26, 27]. Static atomic displacements (SADs) caused by the different covalent radii of the metal atoms were incorporated by force relaxation with the LAMMPS program package [28] employing the empirical Stillinger-Weber type potential proposed by Schowalter et al. [29] for Al-N and Ga-N bonds, while for In-N interactions parametrisation from Ref. [30] was used. The nonuniform sensitivity of the HAADF detector used in the following experiment was also taken into account [15]. The resulting images, a section of which is displayed in Fig. 3a, were evaluated atomic-column wise by averaging over Voronoi cells as described in Ref. [16]. To avoid discrepancies between experimental and simulated intensity distributions caused by slightly different mean concentrations, the intensities were divided by their mean, thereby centering the histograms around 1, as shown in Fig. 3b. This is justified, as the overall spread of intensities is less affected by small concentration changes than the mean intensity.

The resulting intensity ratio distribution is visually in very good agreement with a Gaussian distribution as can be seen in Fig. 3b. This agreement was also statistically tested: A chi-square goodness-of-fit test, which allows testing the hypothesis that the simulated values come from a Gaussian distribution by comparing the relative frequencies of simulation and ideal Gaussian distribution, confirms this hypothesis with a significance of $10 \%$ at all thicknesses. The one-sample Kolmogorov-Smirnov-Lilliefors test [31], which is a modification of the well-known Kolmogorov-Smirnov test taking into account that mean and standard deviation of the Gaussian distribution are not known in advance here, accepts even with $20 \%$ significance.

It shall be stressed here, that the statistical significance represents the probability of erroneously rejecting the tested hypothesis, in this case that the underlying distribution is Gaussian. High significance does not necessarily imply, that the probability to only accept the hypothesis if it is true, which is called the statistical power of the test, is also high, though it is commonly used to 


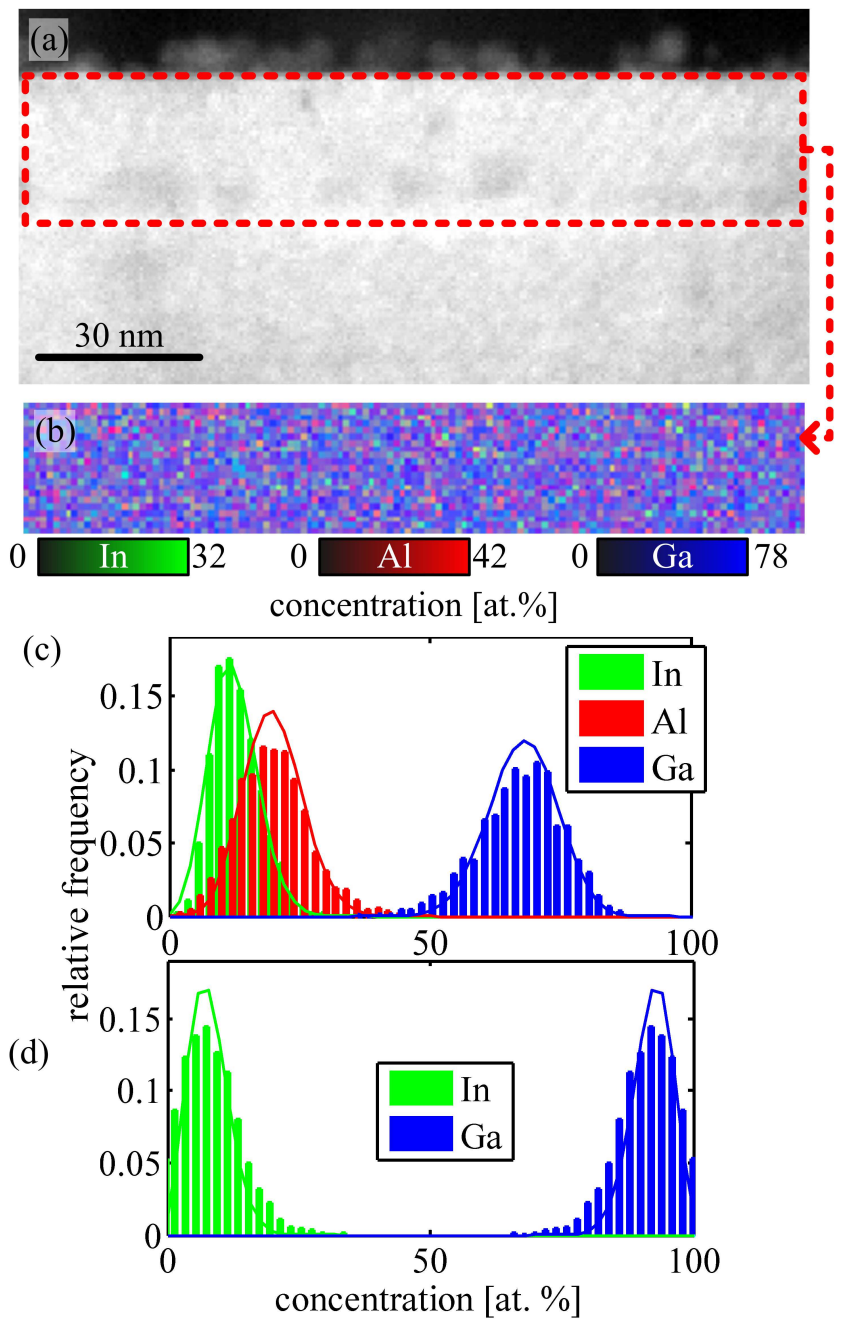

Figure 2: Results of the EDX-investigation: (a) shows an HAADF STEM micrograph of the investigated area with the quaternary layer of composition no.4 marked. The intensity fluctuations are caused by contamination during the long acquisition time of $10 \mathrm{~min}$. The concentration map is displayed color-coded in (b). From this, the histograms of indium, aluminum and gallium concentrations presented in (c) were determined. (d) shows the histograms for the ternary InGaN sample (composition no.1) for comparison. The solid curves show the distributions expected for a homogeneous alloy. 


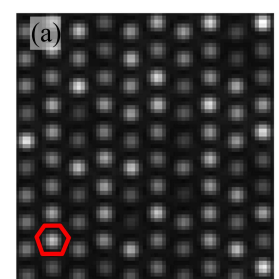

$0 \mathrm{HAAD} 0.02$
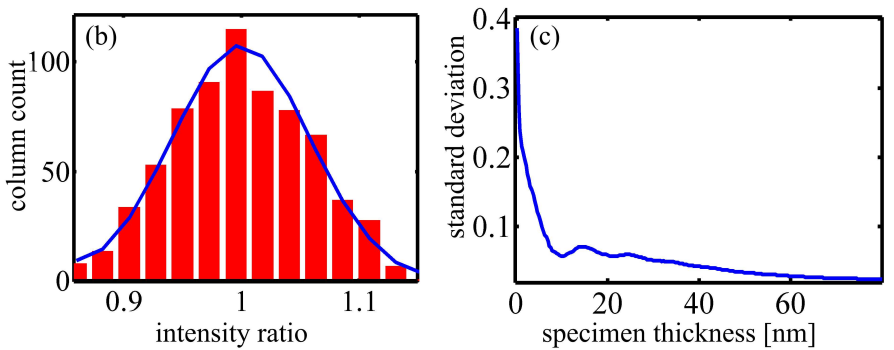

Figure 3: HAADF STEM simulation of a random-alloy AlInGaN crystal with 31 at. $\% \mathrm{Al}$ and 16 at.\% In: In (a), a section of the image simulated for a specimen thickness of $2 \mathrm{~nm}$ is depicted. The red polygon exemplifies a Voronoi cell. Intensities averaged in Voronoi cells yield the histogram displayed in (b). The solid blue curve is the fitted Gaussian distribution. (c) depicts the standard deviation of the intensity ratio plotted vs. specimen thickness.

asses the results of a test. The test power has therefore also be checked which is done later in this section.

The standard deviation of the simulated intensity ratio is plotted in Fig. 3c. For very thin crystals with thicknesses below $10 \mathrm{~nm}$ it is varying rapidly with thickness, but for thicker specimens the variation of the standard deviation is smaller. A local maximum can be found at $\approx 15 \mathrm{~nm}$. The following measurements were hence executed at this thickness to avoid an artificial increase of the obtained standard deviations by an error of the measured specimen thickness.

High-resolution HAADF STEM images were acquired accordingly at a specimen thickness of $(16 \pm 2) \mathrm{nm}$, which was measured from the intensity in the GaN areas. The intensity was normalised to the incident intensity [14-16] and evaluated in the same way as the simulated data before. An exemplary micrograph is shown in Fig. 4a.

In the experiment, the intensity of atom columns varies even in pure GaN. This noise is primarily caused by small thickness variations and surface contamination. In the following, it was assumed that the noise caused by these effects is the same in the quaternary layers. However, it might be even larger in the AlInGaN as selective etching causing stronger thickness variations is more probable there. Therefore, the noise with a standard deviation of 0.03 estimated from a GaN area as the one marked red in Fig. 4b can be considered as a lower bound for AlInGaN.

As standard deviations are added Pythagorean, the relative contribution of this noise to the standard deviation of 0.08 measured in the quaternary material is less than $8 \%$. The suchlike characterised noise was added to the simulated data by adding Gaussian distributed random numbers with the according standard deviation. The resulting histogram is shown in Fig. 4c in red. With the noise added, the simulated data should be fully comparable to the experiment. Hence, the intensity ratio distribution was also determined from the measured intensity of the AlInGaN layer with composition no.4 as marked in green in Fig. 4b, leading to the green histogram in Fig 4c. Apparantly, both distri- 

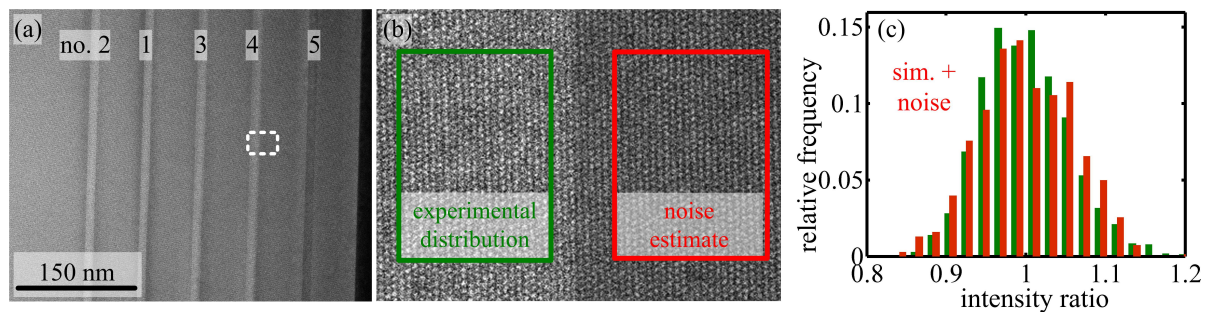

Figure 4: Evaluation of the experimental HAADF STEM images: (a) is an overview of the investigated conventionally prepared specimen. The dotted rectangle marks the area, in which the high-resolution micrograph in (b) was taken. Averaging the image intensity over Voronoi cells yields an experimental noise estimate. (c) shows the experimental histogram from the analysis of the quaternary area marked by the green frame in (b) in direct comparison to the simulation results with incorporated noise marked by the red frame.

butions are similar. This could also be confirmed by various statistical tests: Not only is the one-sample Kolmogorov-Smirnov-Lilliefors test accepting normality of the experimental distribution with $20 \%$ significance, as it did for the simulation.

The two-sample Kolmogorov-Smirnov test, which checks whether two samples are from the same not necessarily Gaussian distribution, also confirms that the STEM intensity in the quaternary material has the same distribution as the noise-incorporated simulation with an exceptional significance of $45 \%$. In a further statistical examination, the identity of the variances of simulation and experiment was also accepted by a two-sample F-Test and Bartlett's test, which allow testing this identity, with $30 \%$ significance. The unity of this test results strongly indicate the identity of the measured HAADF-intensity and the one expected for an entirely random alloy and hence a homogeneous composition in the quaternary material.

However, as mentioned before, despite the very high significance with which the identity of distributions was accepted, the statistical power of the tests could still be small, if cluster-formation did not strongly alter the intensity-ratio distribution. To check against an error of this kind, another STEM simulation with a single cubic cluster with a side length of $3 \mathrm{~nm}$ with increased indium concentration of 40 at.\% and reduced aluminum concentration of 20 at.\% buried inside a supercell of $15 \mathrm{~nm}$ thickness was conducted. Size and composition of this cluster lies well in the range of inhomogeneities reported for AlInGaN [11, 13]. The resulting image was evaluated as described above. The result is shown in Fig. 5 in direct comparison to the random-alloy distribution. These histograms are clearly different and in fact the two-sample Kolmogorov-Smirnov test rejects their identity and the one-sample Kolmogorov-Smirnov-Lilliefors test does not accept Gaussian distribution of the intensity ratio obtained for the simulated cluster, not even for a significance of only $1 \%$. It can thus be concluded, that the statistical analysis of HAADF STEM data presented here powerful enough and hence well-suited to exclude existence of nanometer-scale clusters with an indium concentration increased from approximately 20 at.\% to 40 at.\%. There- 

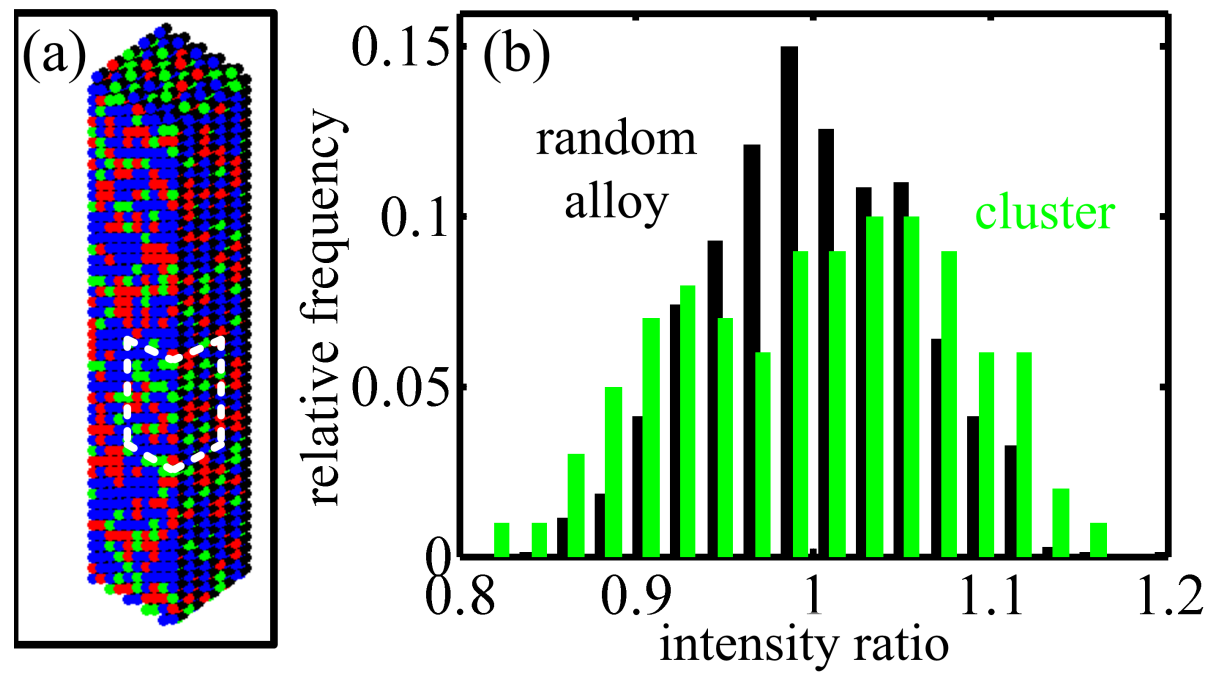

Figure 5: Simulated HAADF STEM imaging of an indium-rich cluster: The supercell shown in (a) contains an indium-rich cluster marked by the dotted frame. The cubic-shaped cluster has a side length of $3 \mathrm{~nm}$, the supercell is $15 \mathrm{~nm}$ high. The resulting histogram is shown in green in (b) in comparison to the distribution of a random alloy in black.

fore, the AlInGaN-layer investigated here is shown to be homogeneous to this extent.

An attempt was also made to determine the indium and aluminum concentrations by simultaneous evaluation of HAADF intensity and lattice strain measured from the high-resolution STEM images as described by Grieb et al. [32]. However, this method turned out to be less applicable for the AlInGaN material system as is illustrated in Fig. 6: Simulations as described above were conducted for various compositions from the two-dimensional concentration space as reference for the intensity. Additionally, the biaxial lattice strain in growth direction was calculated from elasticity theory for comparison with the measured strain. The isolines for both datasets, on which compositions with the same HAADF intensity or the same strain, respectively, lie, are shown for a fixed specimen thickness of $50 \mathrm{~nm}$. This is a common thickness for quantitative HAADF STEM measurements in the AlGaN/InGaN-system [15]. It becomes apparent that these isolines are nearly parallel, meaning that the information which can be gained from the measurement of HAADF intensity and lattice strain is quasi equivalent. Even with both quantities measured the simultaneous determination of both the aluminum and the indium concentration is therefore impossible under experimental conditions as two non-equivalent pieces of information would be necessary for this. Using this method the possible compositional range is thus given by a narrow region along the isoline that corresponds to the measured normalised HAADF intensity.

This concentration range, however, was found to be in full agreement with the other measurements in Tab. 1 as is illustrated in Fig 9. For the ternary 


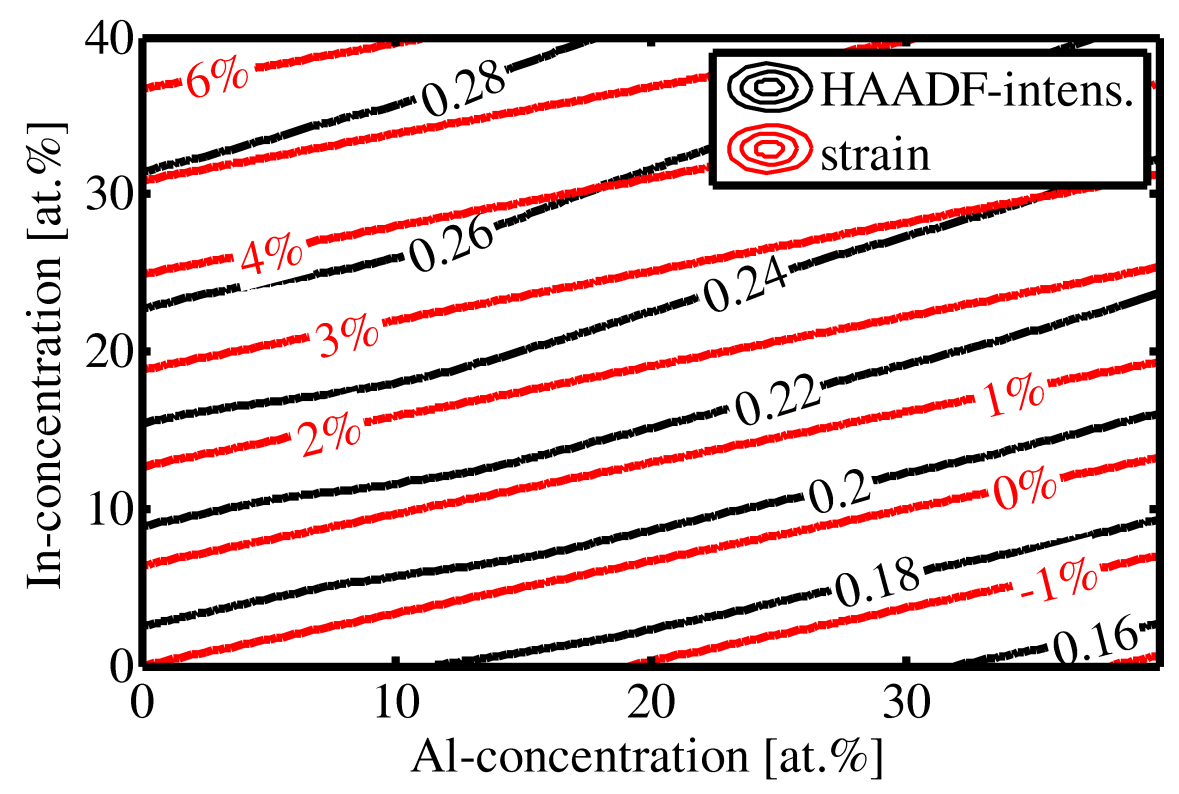

Figure 6: Comparison of HAADF STEM and strain reference data: The isolines of STEM intensity (normalised to the incident probe intensity) simulated with the frozen-lattice method and a specimen thickness of $50 \mathrm{~nm}$ are drawn in black, while the isolines of biaxial strain calculated by elasticity theory are plotted in red versus indium and aluminum concentrations. The red and black isolines run almost in parallel.

InGaN layer (composition no.1), where the prior knowledge of vanishing $\mathrm{Al}$ concentration could be utilised to get an exact concentration, the indium concentration was determined to be $(11 \pm 1)$ at.\% and for the AlGaN layer (composition no.5) $(38 \pm 1)$ at.\% $\mathrm{Al}$ was found, which is in excellent agreement with the other methods.

\subsection{APT-Analysis}

The specimens investigated in the previous section were also examined in a comparative APT study. The measurement conditions were optimised for the material system based on the findings of Mehrtens et al. [33] by cooling down the specimen tip to $20 \mathrm{~K}$ and using a laser-pulse energy of $50 \mathrm{pJ}$ at a pulse frequency of $100 \mathrm{kHz}$ for a laser wavelength of $532 \mathrm{~nm}$ and a pulse length of $\approx 10 \mathrm{ps}$. 60 million ions were detected during the entire measurement. Due to the optimisation of acquisition parameters, a III:N-ratio of 52:48 could be achieved, which is close to the expected 1:1 ratio. The deficiency of measured nitrogen has been reported to be due to the dissociation of complex ions between evaporation from the specimen and detection, causing inaccurate identification of incident ions [33, 34]. Fig. 7a shows an image of the reconstructed specimen. All five AlInGaN layers were detected entirely. The layer thicknesses and distances are in excellent agreement with the STEM micrographs. An atomic-concentration 
profile was extracted by averaging in lateral direction and for a bin width of $0.2 \mathrm{~nm}$ in growth direction, which is presented in Fig. 7b. The average concentration of each layer is also given in Tab. 1 and illustrated in Fig. 9, where fair agreement with the EDX results within the according precisions becomes apparent.

The homogeneity was then statistically investigated similar to the STEM data following Ref. [35]. The volume of each layer was divided into voxels, each containing a fixed number of detected ions. The concentration of each ion species was then calculated and the respective concentration distribution inside each layer was determined. The resulting histograms for Al-, In-, Gaand $\mathrm{N}_{2}$-ions for layer no. 4 and voxels containing 40 ions are displayed in Fig. 7c in comparison with the binomial distribution expected for a random alloy. The visibly good fit between both was tested by the chi-square test and accepted with a significance of $10 \%$ for all detected ion types. The test was repeated for voxel sizes of 20, 100 and 200 ions, respectively, which corresponds to voxels with approximate side lengths from 1.4 to $3.2 \mathrm{~nm}$. It yielded an identity with a random-alloy distribution with more than $5 \%$ significance in all cases. Similar results were obtained for the other layers. Additionally, the radial distribution around indium atoms was investigated by calculating the radially averaged concentration as a function of the distance to a central indium atom and then averaging over all indium atoms. The result is shown in Fig. 7d for layer no. 4: For indium-clustering, one would expect a significant increase in indium concentration for small radial distances, which was not observed. The radial distribution is almost constant. The APT study hence does confirm the previous findings. All layers are found to be homogeneous on various scales.

To check the power of the previous test, a test structure with a single AlInGaN-layer of a similar composition as no.4 was investigated with the same parameters as before. Due to a pulsed MOVPE process, which involved an alternating injection of In-, Al- and Ga-precursors, this sample exhibited a stripy appearance in HAADF STEM images caused by monolayers of alternating different compositions, as is shown in Fig. 8a. The stripes are also clearly visible in the APT reconstruction in Fig. 8b, in which they were identified as fluctuations of the aluminum concentration of approximately \pm 5 at.\% with a simultaneously constant indium content. This clearly inhomogeneous sample was also analysed by testing the concentration distributions against the random-alloy expectation with the chi-square test. The corresponding histograms are shown in Fig. 8c. Random distribution of gallium and aluminum was clearly rejected, while it was accepted for indium and nitrogen with a significance of $20 \%$. The test therefore clearly proves its suitability to identify inhomogeneities caused by fluctuations of the magnitude of 10 at.\% on the sub-nanometer scale.

\section{Summary}

In conclusion, an investigation of the homogeneity of AlInGaN layers of different concentrations by statistical means using EDX, HAADF STEM and APT was presented. The layers were found to exhibit random-alloy distribution with 

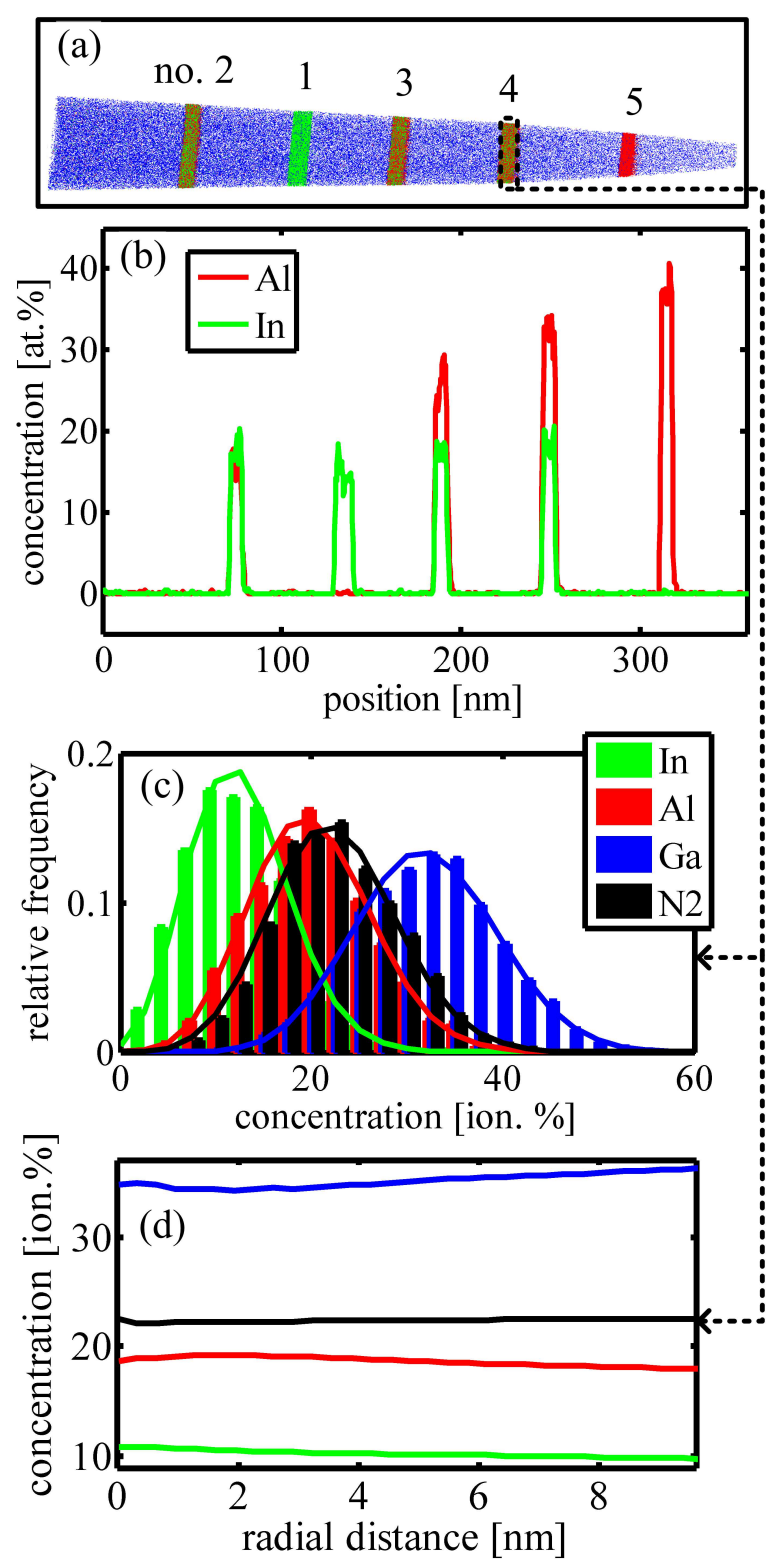

Figure 7: Evaluation of the APT measurement: The reconstruction of the specimen is shown in (a). (b) displays the resulting concentration profile along the growth direction. In (c) the distribution of various detected ions inside a layer (bars) is compared to the random-alloy expectation (solid curves). (d) is a plot of the ionic concentration in dependence of the radial distance from indium ions. Indications for non-random fluctuations are not found. 

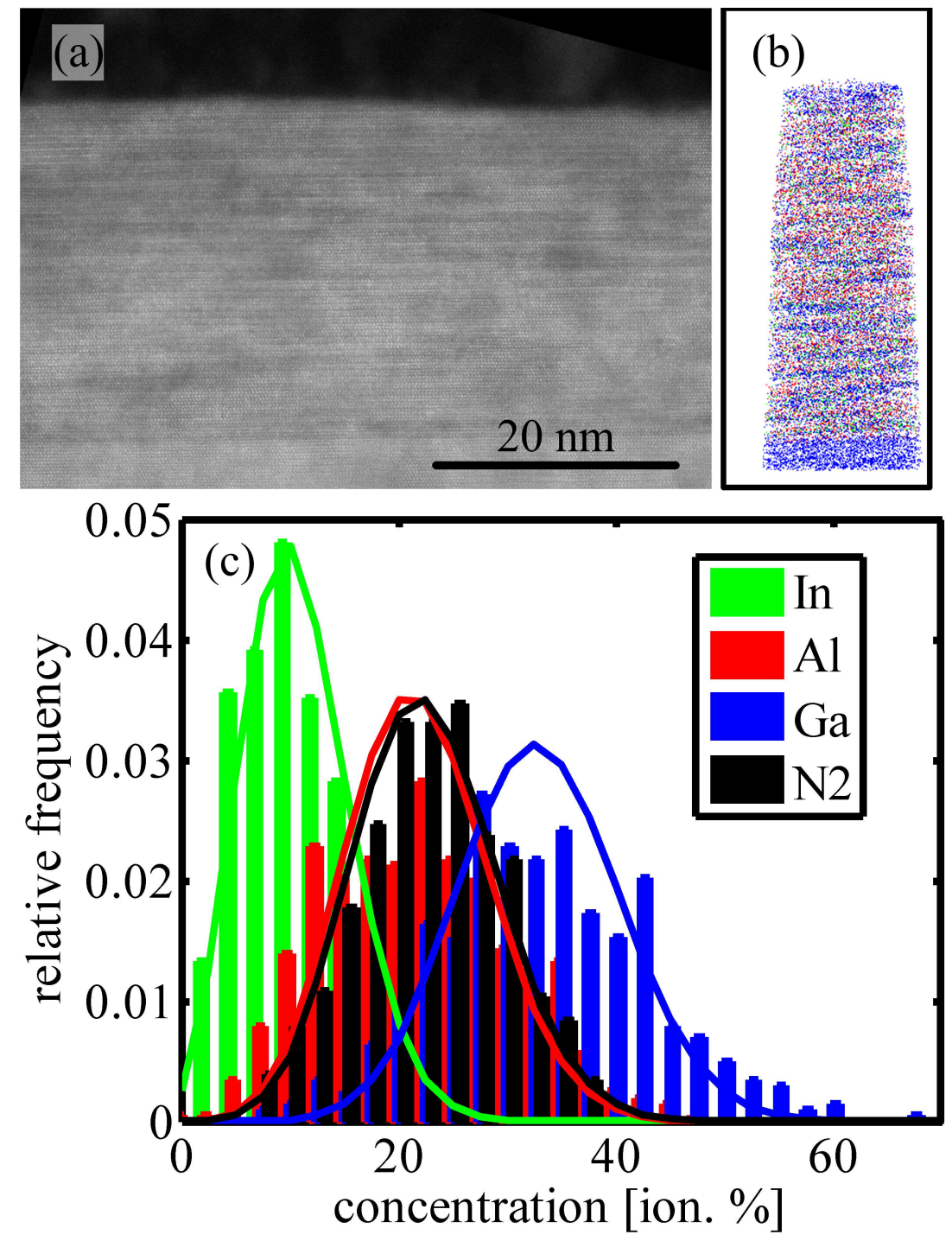

Figure 8: APT evaluation of an inhomogeneous AlInGaN layer: Intensity fluctuations between atomic layers were observable in HAADF STEM micrographs as shown in (a), the reconstruction in (b) revealed them to be caused by varying aluminum and gallium concentrations, which caused distinct deviation of measured (bars) and random-alloy (solid curves) distributions in the histogram (c). 
high significances down to the sub-nanometer scale. The applicability of the tests to the two-dimensional HAADF STEM data was confirmed by additional simulations, in which the statistical power was demonstrated and proved to be comparable to corresponding analyses on three-dimensional APT reconstructions.

The observed increase in width of the PL-emission peak can hence be deducted to stem solely from local concentration fluctuations inherent to a randomalloy distribution of elements. It can be furthermore concluded that, in the studied composition range, the MOVPE parameters used for the growth of the investigated layers do indeed allow epitaxy of homogeneous quaternary AlIn$\mathrm{GaN}$ without detectable phase separation. In respect to the previous reports of indium cluster formation [10-13] this conclusion does not apply to any growth setup in general. Nevertheless it is shown, that homogeneous MOVPE growth of AlInGaN for these concentrations is possible.

Finally, the quaternary concentrations were determined and consistent results were attained as is also illustrated in Fig. 9, where the summarised results of all measurements presented here are seen to coincide within their precisions, though with HAADF STEM, the quaternary composition could not be determined unambiguously, as a second non-equivalent information channel would be needed. Nevertheless, it was demonstrated, that the statistical analysis of STEM micrographs by comparison to simulations is sufficiently powerful to exclude the presence of nm-scale chemical species clustering.

\section{Acknowledgements}

The research leading to these results has received funding from the European Union Seventh Framework Programme under Grant Agreement 312483 - ESTEEM2 (Integrated Infrastructure Initiative I3) and was supported by the Deutsche Forschungsgemeinschaft under contracts RO2057/4-2, RO2057/81 and MU3660/1-1.

[1] H. Hirayama, Quaternary InAlGaN-based high-efficiency ultraviolet lightemitting diodes, Journal of Applied Physics 97 (2005) 091101.

[2] M. F. Schubert, J. Xu, J. K. Kim, E. F. Schubert, M. H. Kim, S. Yoon, S. M. Lee, C. Sone, T. Sakong, Y. Park, Polarization-matched GaInNAlGaInN multi-quantum-well light-emitting diodes with reduced efficiency droop, Applied Physics Letters 93 (2008) 041102.

[3] B. Reuters, A. Wille, N. Ketteniss, H. Hahn, B. Hollnder, M. Heuken, H. Kalisch, A. Vescan, Polarization-Engineered Enhancement-Mode HighElectron-Mobility Transistors Using Quaternary AlInGaN Barrier Layers, Journal of Electronic Materials 42 (2013) 826-832.

[4] T. Lim, R. Aidam, P. Waltereit, T. Henkel, R. Quay, R. Lozar, T. Maier, L. Kirste, O. Ambacher, GaN-Based Submicrometer HEMTs With LatticeMatched InAlGaN Barrier Grown by MBE, Electron Device Letters, IEEE 31 (2010) 671-673. 


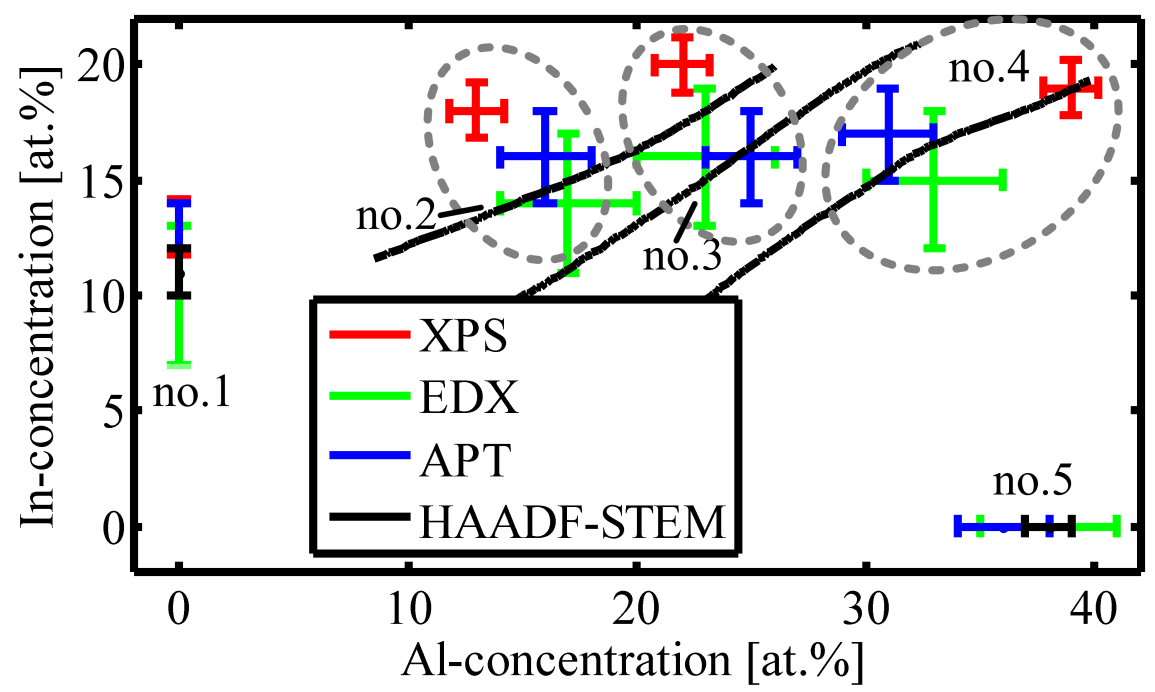

Figure 9: Overview of the measured concentrations: The results of all investigated compositions from the different methods are marked with their respective errors (see Tab. 1). The concentration pairs of the quaternary compositions no.2 to 4 , that are consistent with the measured HAADF STEM intensity are drawn as black isolines (only reasonable part was plotted for better readability). The XPS-data was taken from [20]. The values from EDX, HAADF STEM and APT coincide within their error bars.

[5] P.-M. Tu, C.-Y. Chang, S.-C. Huang, C.-H. Chiu, J.-R. Chang, W.-T. Chang, D.-S. Wuu, H.-W. Zan, C.-C. Lin, H.-C. Kuo, C.-P. Hsu, Investigation of efficiency droop for InGaN-based UV light-emitting diodes with InAlGaN barrier, Applied Physics Letters 98 (2011) 211107.

[6] I. Gorczyca, S. P. Łepkowski, T. Suski, N. E. Christensen, A. Svane, Influence of indium clustering on the band structure of semiconducting ternary and quaternary nitride alloys, Phys. Rev. B 80 (2009) 075202.

[7] I. Gorczyca, A. Kamińska, G. Staszczak, R. Czernecki, S. P. Łepkowski, T. Suski, H. P. D. Schenk, M. Glauser, R. Butté, J.-F. Carlin, E. Feltin, N. Grandjean, N. E. Christensen, A. Svane, Anomalous composition dependence of the band gap pressure coefficients in In-containing nitride semiconductors, Phys. Rev. B 81 (2010) 235206.

[8] I. Gorczyca, T. Suski, N. E. Christensen, A. Svane, Band gap bowing in quaternary nitride semiconducting alloys, Applied Physics Letters 98 (2011) 241905.

[9] Z. Chuan-Zhen, Z. Rong, L. Bin, L. Ming, X. Xiang-Qian, X. Zi-Li, Z. YouDou, A Band-Gap Energy Model of the Quaternary Alloy InGaAlN using Modified Simplified Coherent Potential Approximation, Chinese Physics Letters 30 (2013) 076101. 
[10] C. H. Chen, Y. F. Chen, Z. H. Lan, L. C. Chen, K. H. Chen, H. X. Jiang, J. Y. Lin, Mechanism of enhanced luminescence in InAlGaN quaternary epilayers, Applied Physics Letters 84 (2004) 1480-1482.

[11] J. Wu, J. Li, G. Cong, H. Wei, P. Zhang, W. Hu, X. Liu, Q. Zhu, Z. Wang, Q. Jia, L. Guo, Temperature dependence of the formation of nano-scale indium clusters in InAlGaN alloys on $\mathrm{Si}(111)$ substrates, Nanotechnology 17 (2006) 1251.

[12] T. Matsuoka, Unstable mixing region in wurtzite InGaAlN, Journal of Crystal Growth 189190 (1998) 19 - 23.

[13] M. Marques, L. K. Teles, L. M. R. Scolfaro, L. G. Ferreira, J. R. Leite, Microscopic description of the phase separation process in AlGaInN quaternary alloys, Phys. Rev. B 70 (2004) 073202.

[14] J. M. LeBeau, S. Stemmer, Experimental quantification of annular darkfield images in scanning transmission electron microscopy, Ultramicroscopy 108 (2008) $1653-1658$.

[15] A. Rosenauer, T. Mehrtens, K. Müller, K. Gries, M. Schowalter, P. V. Satyam, S. Bley, C. Tessarek, D. Hommel, K. Sebald, M. Seyfried, J. Gutowski, A. Avramescu, K. Engl, S. Lutgen, Composition mapping in InGaN by scanning transmission electron microscopy, Ultramicroscopy 111 (2011) $1316-1327$.

[16] A. Rosenauer, K. Gries, K. Müller, A. Pretorius, M. Schowalter, A. Avramescu, K. Engl, S. Lutgen, Measurement of specimen thickness and composition in AlGaN/GaN using high-angle annular dark field images, Ultramicroscopy 109 (2009) $1171-1182$.

[17] J. P. O’Neill, I. M. Ross, A. G. Cullis, T. Wang, P. J. Parbrook, Electronbeam-induced segregation in InGaN/GaN multiple-quantum wells, Applied Physics Letters 83 (2003) 1965-1967.

[18] A. J. D'Alfonso, B. Freitag, D. Klenov, L. J. Allen, Atomic-resolution chemical mapping using energy-dispersive x-ray spectroscopy, Phys. Rev. B 81 (2010) 100101.

[19] U. Pohl, Methods of Epitaxy, in: Epitaxy of Semiconductors, Graduate Texts in Physics, Springer Berlin Heidelberg, 2013, pp. 275-313.

[20] J.-P. Ahl, J. Hertkorn, H. Koch, B. Galler, B. Michel, M. Binder, B. Holländer, Morphology, growth mode and indium incorporation of MOVPE grown InGaN and AlInGaN: A comparison, Journal of Crystal Growth 398 (2014) $33-39$.

[21] L. Giannuzzi, F. Stevie, A review of focused ion beam milling techniques for $\{$ TEM $\}$ specimen preparation, Micron 30 (1999) $197-204$. 
[22] T. Mehrtens, S. Bley, P. V. Satyam, A. Rosenauer, Optimization of the preparation of GaN-based specimens with low-energy ion milling for (S)TEM, Micron 43 (2012) $902-909$.

[23] K. Thompson, D. Lawrence, D. Larson, J. Olson, T. Kelly, B. Gorman, In situ site-specific specimen preparation for atom probe tomography, Ultramicroscopy 107 (2007) $131-139$.

[24] G. Cliff, G. W. Lorimer, The quantitative analysis of thin specimens, Journal of Microscopy 103 (1975) 203-207.

[25] A. Rosenauer, M. Schowalter, STEMsim A new software tool for simulation of STEM HAADF Z-contrast imaging, in: A. G. Cullis, P. A. Midgley (Eds.), Springer Proceedings in Physics: Microscopy of Semiconducting Materials Conference 2007, Springer Netherlands, 2007, p. 170172.

[26] D. A. Muller, B. Edwards, E. J. Kirkland, J. Silcox, Simulation of thermal diffuse scattering including a detailed phonon dispersion curve, Ultramicroscopy 86 (2001) 371 - 380. International Symposium on Spectroscopy of Materials.

[27] A. Rosenauer, M. Schowalter, J. T. Titantah, D. Lamoen, An emissionpotential multislice approximation to simulate thermal diffuse scattering in high-resolution transmission electron microscopy, Ultramicroscopy 108 (2008) $1504-1513$.

[28] S. Plimpton, Fast Parallel Algorithms for Short-Range Molecular Dynamics, Journal of Computational Physics 117 (1995) 1 - 19.

[29] M. Schowalter, I. Stoffers, F. F. Krause, T. Mehrtens, K. Müller, M. Fandrich, T. Aschenbrenner, D. Hommel, A. Rosenauer, Influence of Static Atomic Displacements on Composition Quantification of AlGaN/GaN Heterostructures from HAADF-STEM Images, Microscopy and Microanalysis 20 (2014) 1463-1470.

[30] H. Lei, J. Chen, S. Petit, P. Ruterana, X. Jiang, G. Nouet, StillingerWeber parameters for In and N atoms, Superlattices and Microstructures 40 (2006) 464 - 469. E-MRS 2006 Symposium S: Material Science and Technology of Wide Bandgap Semiconductors 2006 Spring Meeting of the European Materials Research Society.

[31] H. W. Lilliefors, On the Kolmogorov-Smirnov Test for Normality with Mean and Variance Unknown, Journal of the American Statistical Association 62 (1967) 399-402.

[32] T. Grieb, K. Müller, E. Cadel, A. Beyer, M. Schowalter, E. Talbot, K. Volz, A. Rosenauer, Simultaneous Quantification of Indium and Nitrogen Concentration in InGaNAs Using HAADF-STEM, Microscopy and Microanalysis 20 (2014) $1740-1752$. 
[33] T. Mehrtens, M. Schowalter, D. Tytko, P. Choi, D. Raabe, L. Hoffmann, H. Jönen, U. Rossow, A. Hangleiter, A. Rosenauer, Measurement of the indium concentration in high indium content InGaN layers by scanning transmission electron microscopy and atom probe tomography, Applied Physics Letters 102 (2013) 132112.

[34] D. Saxey, Correlated ion analysis and the interpretation of atom probe mass spectra, Ultramicroscopy 111 (2011) 473 - 479. Special Issue: 52nd International Field Emission Symposium.

[35] M. P. Moody, L. T. Stephenson, A. V. Ceguerra, S. P. Ringer, Quantitative binomial distribution analyses of nanoscale like-solute atom clustering and segregation in atom probe tomography data, Microscopy Research and Technique 71 (2008) 542-550. 